\title{
The effects of estrogen on temporal and numerical processing in ovariectomized female rats
}

\author{
LORI ROSS and ANGELO SANTI \\ Wilfrid Laurier University, Waterloo, Ontario, Canada
}

\begin{abstract}
Ovariectomized rats were trained to discriminate discrete sound sequences that varied in time or number. On time trials, the number of sounds was held constant at four, and the duration of the sound sequences was either 2 or 8 sec. On number trials, the duration of the sound sequence was a constant $4 \mathrm{sec}$, and the number of sounds was two or eight. After reaching criterion, half the rats received injections of $17-\beta$-estradiol 3-benzoate ( $8 \mu \mathrm{g}$ per $\mathrm{kg}$ bodyweight) for a period of 2 weeks. In Experiment 1 , estradiol treatment significantly decreased both the number of training trials completed and discrimination accuracy. For the ovariectomized control rats, the psychophysical functions for time and number had properties that were consistent with those in the previous literature. For the ovariectomized estradiol-treated rats, the psychophysical functions were initially flatter, but as time since estradiol exposure increased, they approached those of control rats. The points of subjective equality were equivalent for estradiol-treated and control rats, and both groups performed with higher accuracy on time trials than on number trials. In Experiment 2, prefeeding the rats before a session decreased the number of trials completed but had no significant effect on discrimination accuracy. Therefore, estradiol's effect on the accuracy of time and number discriminations in Experiment 1 was not due to its effect on food motivation. These findings are discussed in terms of the internal clock model, their relation to studies of spatial processing, and implications for human health.
\end{abstract}

Although estrogen has long been implicated in reproductive function, relatively little is understood about actions of estrogen that do not seem to be directly linked to reproduction, and in particular, those that may involve the central nervous system. In humans, for example, endogenous estrogen levels are thought to influence verbal fluency, spatial tasks, verbal memory tests, fine motor skills, and coordination of movement in women (McEwen, Alves, Bulloch, \& Weiland, 1997). In addition, preliminary reports suggest that estrogen replacement therapy can slow the cognitive decline associated with Alzheimer's disease in postmenopausal women (Fillit, 1994; Gibbs \& Aggarwal, 1998; Henderson, 1997). The mechanisms involved in this connection between estradiol and cognitive processing are not well understood, although both genomic and nongenomic mechanisms have been postulated (Fink, Sumner, McQueen, Wilson, \& Rosie, 1998; Packard, 1998).

Much of the research relating estrogen to cognition in animals has focused on tests of spatial processing. Daniel, Fader, Spencer, and Dohanich (1997) found that ovariec-

This research was supported by a grant from the Natural Sciences and Engineering Research Council of Canada (OGPOOD6378) to A.S., and by a grant from the STEP Council of Wilfrid Laurier University to L.R. The authors thank Marion Corrick and David Hemsworth for their technical assistance and Melanie Bucking for assisting with some of the data collection in Experiment 2. L.R. is currently at the Institute of Medical Sciences, University of Toronto (l.ross@utoronto.ca). Correspondence should be addressed to A. Santi, Department of Psychology, Wilfrid Laurier University, Waterloo, ON, N2L 3C5 Canada (e-mail: asanti@wlu.ca). tomized (OVX) females given estradiol via Silastic capsules prior to training on a radial arm maze task, during acquisition, or both, had improved performance relative to OVX controls. Luine, Richards, Wu, and Beck (1998) also found that performance during acquisition of an eightarm maze task was better in estrogen-treated OVX rats than in control OVX rats, but only after several days of estrogen treatment. Posttraining injections of estradiol, given either centrally or peripherally, have also been found to enhance memory on a hidden platform water maze task (Packard \& Teather, 1997a, 1997b). Although there are inconsistencies in the literature (see Dohanich, Fader, \& Javorsky, 1994; Singh, Meyer, Millard, \& Simpkins, 1994), most studies of OVX animals suggest that estradiol facilitates performance on spatial tasks. However, studies of the role of estradiol in naturally cycling rats have yielded less conclusive results (Berry, McMahan, \& Gallagher, 1997; Stackman, Blasberg, Langan, \& Clark, 1997; Warren \& Juraska, 1997).

Physiological evidence suggests that estrogen could be influencing spatial learning through an interaction with the cholinergic system. Gibbs (1997) found that OVX rats given injections of 2,10 , or $25 \mu \mathrm{g}$ estradiol show a doserelated increase in choline acetyltransferase (ChAT)-like immunoreactive cells in the basal forebrain. Singh et al. (1994) found that OVX reduced both high-affinity choline uptake and the activity of ChAT; both effects could be reversed by estradiol administration. Behavioral studies have also related estrogen to cholinergic function: Estradiol can reverse the impairment in spatial performance that results from injection of scopolamine, a cholin- 
ergic antagonist (Dohanich et al., 1994; Fader, Hendricson, \& Dohanich, 1998; Gibbs, Burke, \& Johnson, 1998).

Estrogen is also associated with changes in dendritic spine density in the hippocampus, with up to a $30 \%$ fluctuation in pyramidal neurons across the estrous cycle (Woolley, Gould, Frankfurt, \& McEwen, 1990). While numerous researchers have attempted to relate these estrogen-induced changes in hippocampal connectivity with performance on hippocampal-dependent spatial tasks, the results of these efforts have been difficult to interpret. Some researchers have reported facilitation, while others have reported a disruption in performance or no effect (Berry et al., 1997; Stackman et al., 1997; Warren \& Juraska, 1997). As Woolley (1998) has recently noted, understanding the behavioral consequences of estradiol effects on hormone-induced hippocampal changes may require an examination of the hippocampus's cognitive processing functions with respect to alternative biologically relevant behaviors. Time and number represent stimulus attributes, which, in addition to space, are very important for survival and are richly represented in a wide variety of species (Gallistel, 1990). To date, there have been no published studies on the effects of estrogen on temporal and numerical processing in animals.

According to the internal clock model (Church, 1984), animals time biologically important events using a twostage information processing mechanism. The first stage, the "clock," consists of a pacemaker, a switch, and an accumulator, while the second stage involves working memory and reference memory components. Clock speed is thought to be regulated by dopaminergic activity in the basal ganglia, while cholinergic activity in the frontal cortex has been implicated in the memory stage (Gibbon, Malapani, Dale, \& Gallistel, 1997; Meck, 1996). Much experimental evidence has been generated to suggest that counting employs a similar internal mechanism, with the switch acting in an "event" mode rather than in the "run" and "stop" modes necessary for timing (Breukelaar \& Dalrymple-Alford, 1998, 1999; Meck \& Church, 1983; Meck, Church, \& Gibbon, 1985).

If the observed positive effects of estradiol on spatial tasks are due to a general enhancement of cognitive functioning, then it is reasonable to expect similar effects on nonspatial tasks that require similar cognitive processes. For example, one might expect that estradiol could have a general positive effect on overall accuracy of discriminating time and number. However, it is also important to consider potential effects on the perception of time and number. It is possible that estradiol could influence discrimination accuracy in a general way, while also altering the perception of time and number. Effects on discrimination accuracy would be reflected in vertical shifts of the psychophysical functions for time and number, while effects on perception would be reflected in horizontal shifts of the functions. It is possible that estrogen could speed up or slow down the perception of time with- out necessarily affecting accuracy. In this case, only horizontal shifts of the psychophysical functions for time and number would be expected. This experiment was designed to determine which, if any, of these types of effects estrogen would exert on a time/number discrimination task.

\section{EXPERIMENT 1}

Ovariectomized rats were trained to discriminate discrete sound sequences that varied in time or number. On time trials, the number of sounds was held constant at four, and the duration of the sound sequences was either 2 or $8 \mathrm{sec}$. On number trials, the duration of the sound sequence was a constant $4 \mathrm{sec}$, and the number of sounds was two or eight. After reaching criterion, half the rats received injections of $17 \beta$-estradiol 3-benzoate $(8 \mu \mathrm{g}$ per $\mathrm{kg}$ bodyweight) for a period of 2 weeks. The pattern of estrogen treatment was based on that in previous studies (Gibbs, 1997; Gibbs et al., 1998; Luine et al., 1998), and it was designed to optimize the effects of estrogen on ChAT-like immunoreactive cells in the basal forebrain. In order to allow for optimal effects of estrogen, all rats underwent psychophysical testing to measure the proportion of long/many responses made for time intervals of $2,3,4,5,6$, and $8 \mathrm{sec}$, and for two, three, four, five, six, and eight signal presentations upon completion of the 2week injection period.

\section{Method}

\section{Subjects}

Twelve experimentally naive Long-Evans hooded female rats were used as subjects. The rats arrived from Charles River Canada (St. Constant, PQ) aged 39-43 days and had been ovariectomized by the supplier approximately 1 week before shipping. They were singly housed in clear Plexiglas shoebox cages in a temperatureand humidity-controlled holding room under a 12:12-h light:dark cycle (fluorescent lights on at 7 a.m.). Experimental sessions were conducted during the light phase. No male rats were housed in the same room or run in the same operant chambers for the duration of this experiment. The rats were fed Purina Rat Chow shortly after the experimental session each day in order to maintain bodyweight at between $85 \%$ and $90 \%$ of their free-feeding weights. Animals had access to water at all times in the colony room.

\section{Apparatus}

Four Coulbourn operant chambers (Model E10-10), individually housed in isolation chambers (Model E10-20) and equipped with baffled exhaust fans, were used. On the front wall of the chamber, two retractable levers (Model E23-07 in two of the boxes and Model E23-17 in the other two) were positioned on either side of a pellet feeder (Model E14-06) approximately $3 \mathrm{~cm}$ from the grid floor and $14 \mathrm{~cm}$ apart. Above each lever was a $2.9-\mathrm{kHz}$ Sonalert tone module (Model E 12-02). The pellet feeder was placed in the center of the front wall with the opening approximately $3 \mathrm{~cm}$ from the floor of the chamber and provided access to $45-\mathrm{mg}$ pellets (Bioserve Universal Research Test Diets grain-based rodent pellets). A house light (Model E1 1-01, bulb SL 1819x) positioned $6.5 \mathrm{~cm}$ directly above the pellet feeder and reflecting toward the ceiling of the chamber remained on for the duration of the experimental sessions. 
Onset of both Sonalerts was the carrier of the time and number signals. All experimental events and response measures were controlled by a microcomputer located in the same room.

\section{Procedure}

Pretraining. In order to familiarize the rats with the test chambers and the leverpressing procedure, the animals received 22 pretraining sessions on consecutive days. The rats were placed in the chamber with both the left and right levers retracted. Each trial commenced with the entry of the left or right lever into the chamber. The lever remained extended until it was pressed or $60 \mathrm{sec}$ had elapsed, whichever occurred first. Either event resulted in delivery of a food pellet and retraction of the lever. Pellet delivery produced an audible "click" and the light in the magazine was illuminated for $0.5 \mathrm{sec}$. Each session consisted of 60 reinforced trials. Rats that did not adequately acquire the barpressing response were manually shaped to leverpress. Once leverpressing was established, discrimination training began.

Training. Training sessions consisted of 160 trials (80 timerelevant trials and 80 number-relevant trials) or were $120 \mathrm{~min}$ in length, whichever occurred first. Both time and number trials were composed of repeating tone-on/tone-off cycles. Time-relevant trials consisted of four tone cycles presented for either $2 \mathrm{sec}$ (short) or $8 \mathrm{sec}$ (long). Number-relevant trials were $4 \mathrm{sec}$ in duration and consisted of either two tone cycles (few) or eight tone cycles (many). Each block of four trials consisted of one of each of the four trial types (short, long, few, many) presented in random order. For half of the rats $(n=6)$, a response to the left lever on short/few trials and to the right lever on long/many trials was correct and reinforced with delivery of a food pellet. For the other 6 rats, the reward contingencies were reversed. If a correct response was made, the trial was followed by an intertrial interval of $5,10,15,20$, or $25 \mathrm{sec}$, determined randomly for each trial. After an incorrect response, the levers were retracted. Following a 5 -sec pause, the same trial configuration was repeated (correction trial); however, only the initial noncorrection trial was used in calculating accuracy.

After 15 sessions of training, it was noted that a number of rats (both those that would eventually be assigned to the estradiol group and those that would be assigned to the control group) were not completing 160 trials before the 2 -h time limit had passed. In an attempt to keep the rats working on the discrimination task, the following changes were made on Session 16: If the rat did not press one of the levers within $10 \mathrm{sec}$ of presentation, the trial was repeated (as for a correction trial) until a response was made. Also, after three consecutive incorrect responses had been made on any given trial, the intertrial interval (ITI) was presented and the next trial was initiated. Run times ranged between $55 \mathrm{~min}$ and $2 \mathrm{~h}$, and animals were run 5 days per week. Training continued until criterion of $75 \%$ accuracy on both time-relevant and number-relevant trials was reached; this resulted in a total of 49 training sessions.

\section{Estrogen Injections}

On the morning of the 50th training session, estradiol $(n=6)$ or control $(n=6)$ treatments began. Rats in the estrogen replacement condition received subcutaneous injections of $17 \beta$-estradiol 3 -benzoate on alternate days for a period of 2 weeks. Each injection consisted of $0.4 \mathrm{cc}$ sesame oil with $8 \mu \mathrm{g}$ estradiol benzoate per $\mathrm{kg}$ bodyweight. This pattern of administration has been shown to result in serum estradiol levels of approximately $14.0 \mathrm{pg} / \mathrm{ml}$, which is in the low physiological range (Gibbs, 1997; Gibbs et al., 1998). Control animals received subcutaneous injections of $0.4 \mathrm{cc}$ sesame oil vehicle per $\mathrm{kg}$ bodyweight. It should be noted that this volume (approximately $0.1 \mathrm{cc}$ for a $250-\mathrm{g}$ rat) is similar to that used in Gibbs (1997). Throughout the 14-day injection period, the animals continued baseline training; however, during this period, the animals were run 7 days per week.

Psychophysical testing. Exactly 2 weeks after the first injection, psychophysical testing began and no further injections were given. Animals retained the assignment to the left or right lever on short/few trials that they learned during the training sessions. Testing sessions were also composed of 160 trials: 20 trials for each of the four training stimuli (durations of 2 or $8 \mathrm{sec}$ and frequencies of two or eight events), and 10 trials for each of the intermediate test stimuli $(3,4,5$, or $6 \mathrm{sec}$ or signal frequencies). Responses were reinforced only on trials composed of the four training stimuli. Responses to the intermediate test stimuli were not reinforced. All other conditions were identical to those of the training sessions. During psychophysical testing, animals were run 6 days per week, and a total of 20 test sessions were conducted.

\section{Results and Discussion}

The data obtained for accuracy on the time and number training stimuli (i.e., $2 \mathrm{sec}, 8 \mathrm{sec}$, two signals, eight signals) for both the estradiol group (top panel) and the control group (bottom panel) are presented in Figure 1. The data were organized into four phases of 7 days each. The first phase was composed of the 7 days immediately prior to estradiol/sesame oil injections; the second phase was composed of Injection Days 1-7; the third phase was composed of Injection Days 8-14; and the fourth phase was composed of the 7 days immediately following the last injection (i.e., the first 7 days of psychophysical testing). Across all conditions, the accuracy of time discriminations was greater than that for number discriminations in both groups. In addition, the accuracy of performance in the estradiol group exhibited the greatest decline between the 1 st and the 2 nd weeks of injections (Phases 2 and 3). However, the accuracy of performance in the control group remained high across all phases. An analysis of variance (ANOVA) of these data, with group as the between-subjects factor and dimension (time vs. number) and phase as within-subjects factors, was conducted. The ANOVA revealed significant main effects of group $[F(1,10)=51.52, p<.01]$ and phase $[F(3,30)=17.94$, $p<.01]$, as well as a significant interaction of group and phase $[F(3,30)=12.49, p<.01]$. There was a statistically significant phase effect for the estradiol $[F(3,30)=$ $29.07, p<.001]$, but not for the control group $(F<2)$. This shows that accuracy for both time and number declined across phases in the estradiol group as a result of the estradiol injections. In addition, while accuracy was higher for the control group than for the estradiol group across all phases, the effect was strongest during Phases 2,3 , and $4[F \mathrm{~s}(1,10)=6.74,22.62,49.23$, and 37.27 , respectively]. The analysis also revealed a significant main effect of dimension $[F(1,10)=109.53, p<.01]$, as a result of better performance on time trials than on number trials. The dimension $\times$ phase interaction was also significant $[F(3,30)=4.27, p<.05]$ since accuracy on time and number trials differed most significantly during the postinjection phase (i.e., the first 7 days of psychophys- 

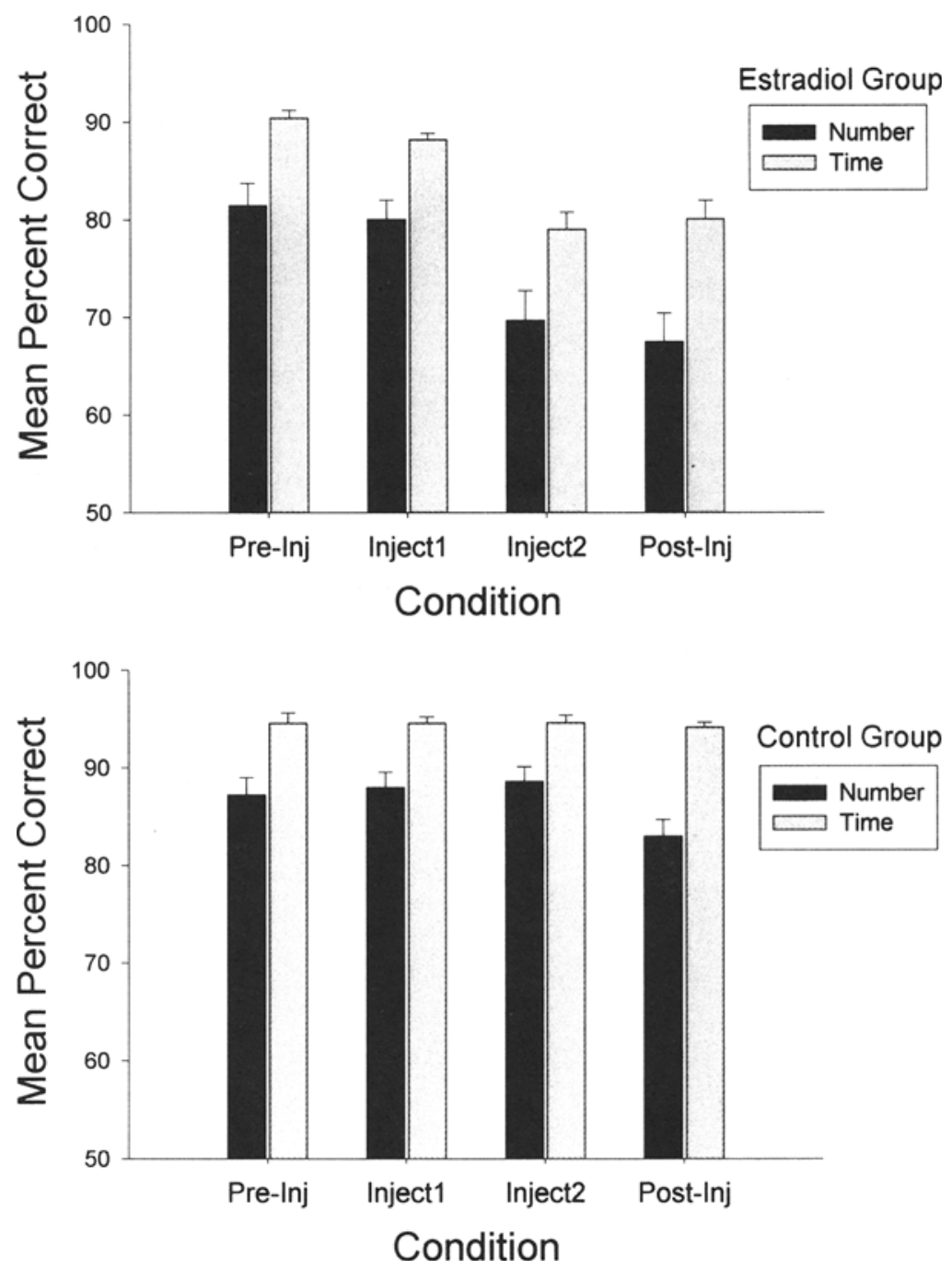

Figure 1. The mean percentage correct on training signal trials for the time and the number dimensions as a function of group. The data for the estradiaol group (top panel) and the control group (bottom panel) are presented for the seven sessions preinjection (Pre-Inj), the first seven sessions of injections (Inject1), the second seven sessions of injection (Inject2), and the seven sessions postinjection (PostInj). Error bars represent standard errors of the mean.

ical testing). Neither the group $\times$ dimension nor the three-way interaction was significant $(F \mathrm{~s}<2)$.

The number of trials completed per session for the animals in each group across the four phases was also computed, and the data are presented in Table 1. The mean number of trials completed by the control group generally increased across phases, essentially reaching the maximum of 160 trials by the last phase. However, the mean number of trials completed by the estradiol group decreased to a minimum during the 2 nd week of estradiol administration and then increased during the postinjection week. These data were analyzed using a mixed design ANOVA with group as the between-subjects factor and phase as the within-subjects factor. There was a significant main effect of group $[F(1,10)=16.93, p<$ $.01]$, a main effect of phase $[F(3,30)=35.69, p<.01]$, and a significant group $\times$ phase interaction $[F(3,30)=$ $35.80, p<.01]$. The estradiol rats completed significantly fewer trials than the control rats during the 2 weeks of injections and the postinjection week $[F(1,10)=$ $8.07,49.64$, and 40.29 , respectively, $p<.05]$.

The psychophysical functions for time and number are shown in Figures 2 and 3, respectively. The mean proportion of long responses is shown in Figure 2 as a function of signal duration averaged over four blocks of five test sessions for the estradiol group, and averaged over all 20 test sessions for the control group. The mean proportion of many responses as a function of signal fre- 
Table 1

Mean Number of Trials Completed per Session Across Phases for Estradiol and Control Groups

\begin{tabular}{lcr}
\multicolumn{1}{c}{ Condition } & $M$ & \multicolumn{1}{c}{$S E$} \\
\hline & Estradiol & \\
Preinjection (Phase 1) & 138.5 & 9.74 \\
Injection 1 (Phase 2) & $112.8^{*}$ & 12.35 \\
Injection 2(Phase 3) & $64.1 \dagger$ & 10.29 \\
Postinjection (Phase 4) & $123.1 \dagger$ & 5.74 \\
& & \\
& Control & \\
Preinjection (Phase 1) & 142.2 & 5.54 \\
Injection 1 (Phase 2) & $150.7^{*}$ & 5.05 \\
Injection 2 (Phase 3) & $148.0 \dagger$ & 6 \\
Postinjection (Phase 4) & $159.6 \dagger$ & 0.29 \\
\hline
\end{tabular}

*Significant difference between the estradiol and control groups $(p<$ $.05)$. Tignificant difference between the estradiol and control groups $(p<.01)$.

quency is presented in Figure 3 averaged in a similar fashion. For both the time and number dimension, the percentage of long/many responses increased as a function of signal value. Both the estradiol and the control group performed more accurately on the time dimension than on the number dimension, as evidenced by the steeper slopes of the time functions. Finally, for both the time and the number dimensions, the psychophysical functions of the estradiol group were flatter during the initial block of testing, but they approached those of the control rats in the later blocks of testing.

An ANOVA of these data, with group as a betweensubjects factor and dimension (time vs. number), block, and signal value as within-subjects factors, was conducted. There was no significant main effect of group $(F=2.03)$ or dimension $(F<1)$, but there was a significant main effect of signal $[F(5,50)=393.23, p<.001)$, indicating that for both the time and number dimensions in both the estradiol and control groups, the percentage of long/many responses increased as a function of signal value. There was a main effect of block $[F(3,30)=2.93$, $p<.05]$, indicating that performance of both groups improved slightly with repeated testing. There were significant two-way interactions of group $\times$ block $[F(3,30)=$ $3.07, p<.05]$, group $\times$ signal $[F(5,50)=7.10, p<.01]$, and most importantly, a significant three-way interaction of group $\times$ block $\times$ signal $[F(15,150)=2.74, p<.01]$. The three-way interaction occurred because the block $x$ signal interaction was significant for the estradiol group $[F(15,150)=4.31, p<.001]$, but not for the control group $(F<1)$. In addition, during the first block of testing, the estradiol and the control group differed significantly at signal values of $2,5,6$, and $8\left[F_{\mathrm{S}}(1,10)=22.90\right.$, $18.5,5.37$, and 16.14 , respectively, $p<.05]$. This reflects the flatter psychophysical function for the estradiol group during the first block of testing. However, by the fourth block of testing, there was no significant difference between the two groups at any signal value. None of the interactions involving group and dimension were statistically significant. The only statistically significant effect associated with dimension was the dimension $\times$ signal interaction $[F(5,50)=39.75, p<.01]$, indicative of better performance on the time dimension than on the number dimension. At signal values less than 4 , the number

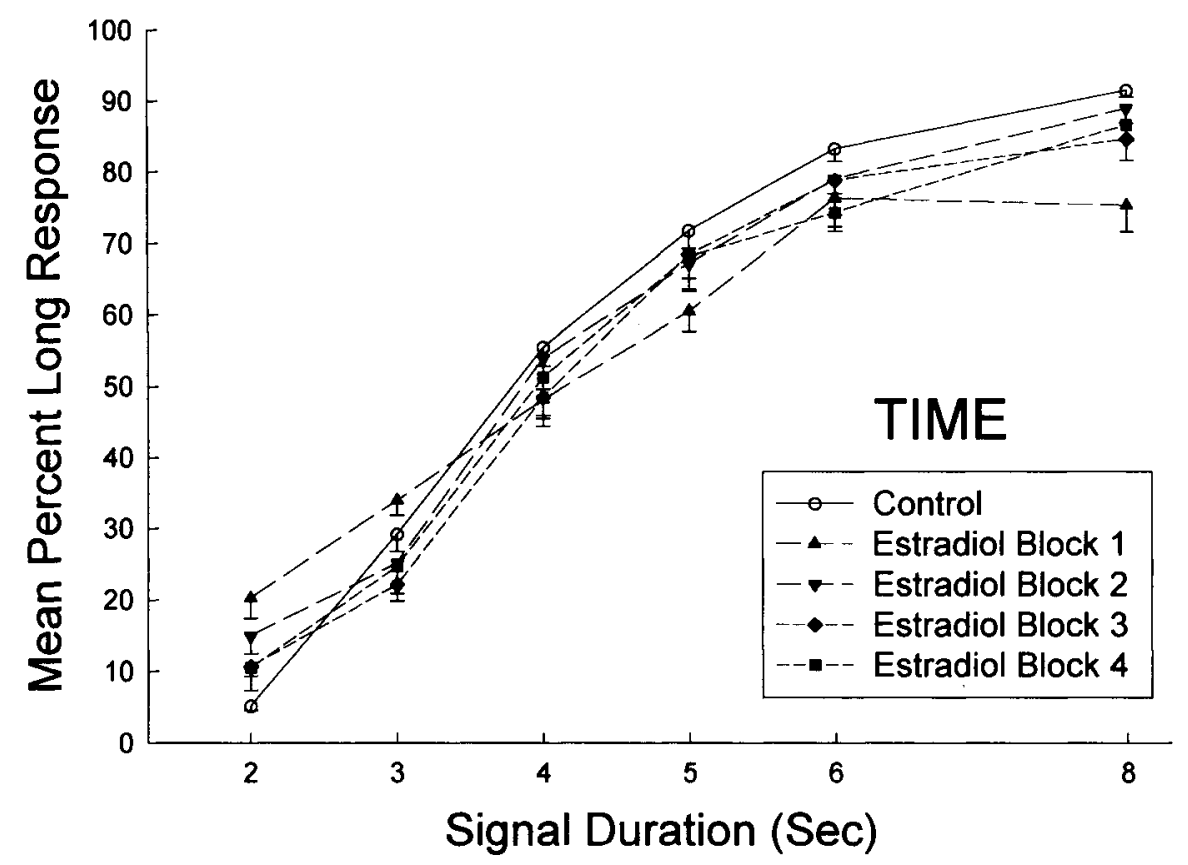

Figure 2. The mean percentage of long responses as a function of signal duration averaged over 20 test sessions for the control group and averaged over five-session blocks for the estradiol group. Error bars represent standard errors of the mean. 


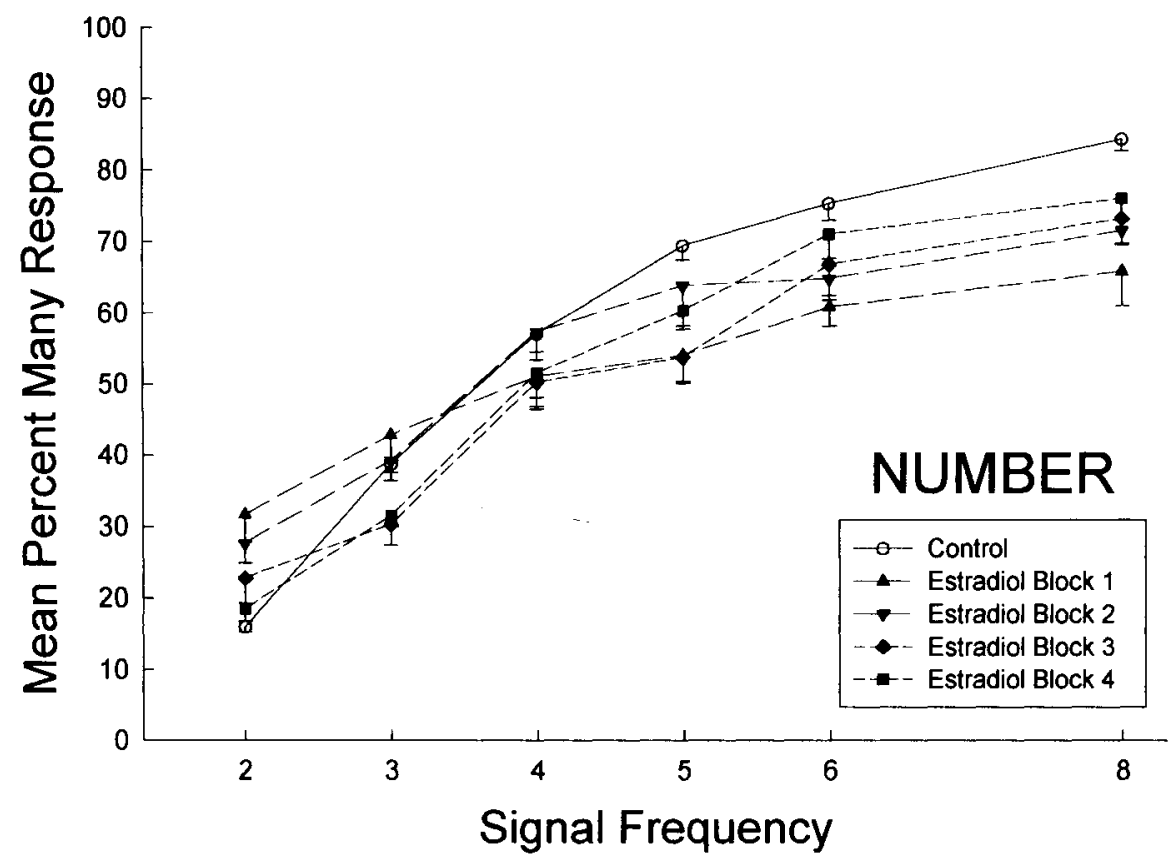

Figure 3. The mean percentage of many responses as a function of signal frequency averaged over 20 test sessions for the control group and averaged over five-session blocks for the estradiol group. Error bars represent standard errors of the mean.

of many responses was significantly greater than the number of long responses $\left[F_{\mathrm{S}}(1,10)=130.33\right.$ and 25.18 , $p<.05$ ], while at signal values greater than 4 , the number of many responses was significantly lower than the number of long responses $[F \mathrm{~s}(1,10)=14.03,60.88,35.99$, $p<.05]$.

The point of subjective equality (PSE) was estimated from the psychophysical function for each rat by conducting linear regressions of the proportion of long and the proportion of many responses for each of three adjacent points in the two functions (time and number). For each rat, the regression equation with the greatest slope for each dimension was used to estimate the PSE by calculating the signal duration or the signal frequency asso- ciated with $50 \%$ of the long or $50 \%$ of the many responses. The regression equations were also used to calculate the difference limen (DL), which represents the average difference between the signal duration or frequency associated with $75 \%$ long or many responses and the signal duration or frequency associated with $25 \%$ long or many responses. Finally, the Weber fraction (WF) was calculated as the DL/PSE. The mean PSEs, DLs, and WFs for each block and each group are given in Table 2. PSEs, DLs, and WFs were analyzed using mixed design ANOVAs (group $\times$ dimension $\times$ block).

PSEs did not differ significantly between groups or across blocks $(F \mathrm{~S}<2)$. However, there was a significant effect of dimension $[F(1,10)=12.52, p<.05]$ indicating

Table 2

Points of Subjective Equality (PSEs), Difference Limens (DLs), and Weber Fractions (WFs) for Time and Number Across Blocks During Psychophysical Testing in the Estradiol and Control Groups

\begin{tabular}{|c|c|c|c|c|c|c|c|c|c|c|c|c|}
\hline \multirow[b]{3}{*}{ Condition } & \multicolumn{4}{|c|}{ PSE } & \multicolumn{4}{|c|}{ DL } & \multicolumn{4}{|c|}{ WF } \\
\hline & \multicolumn{2}{|c|}{ Number } & \multicolumn{2}{|c|}{ Time } & \multicolumn{2}{|c|}{ Number } & \multicolumn{2}{|c|}{ Time } & \multicolumn{2}{|c|}{ Number } & \multicolumn{2}{|c|}{ Time } \\
\hline & $M$ & $S E$ & $M$ & $S E$ & $M$ & $S E$ & $M$ & $S E$ & $M$ & $S E$ & $M$ & $S E$ \\
\hline \multicolumn{13}{|c|}{ Estradiol } \\
\hline Block 1 & 4.14 & 0.27 & 4.21 & 0.16 & 2.3 & 0.29 & 1.55 & 0.11 & 0.55 & 0.1 & 0.37 & 0.02 \\
\hline Block 2 & 3.62 & 0.22 & 3.98 & 0.17 & 1.7 & 0.22 & 1.12 & 0.11 & 0.47 & 0.1 & 0.28 & 0.03 \\
\hline Block 3 & 4.18 & 0.29 & 4.14 & 0.13 & 1.73 & 0.13 & 1.11 & 0.13 & 0.42 & 0 & 0.27 & 0.03 \\
\hline Block 4 & 4.01 & 0.19 & 4.12 & 0.18 & 1.39 & 0.12 & 1.11 & 0.18 & 0.34 & 0 & 0.26 & 0.03 \\
\hline \multicolumn{13}{|c|}{ Control } \\
\hline Block 1 & 3.46 & 0.17 & 3.79 & 0.15 & 1.18 & 0.14 & 0.91 & 0.06 & 0.34 & 0 & 0.24 & 0.01 \\
\hline Block 2 & 3.76 & 0.26 & 3.91 & 0.23 & 1.18 & 0.15 & 0.91 & 0.11 & 0.31 & 0 & 0.23 & 0.03 \\
\hline Block 3 & 3.6 & 0.15 & 3.96 & 0.19 & 1.2 & 0.07 & 1.05 & 0.08 & 0.33 & 0 & 0.26 & 0.01 \\
\hline Block 4 & 4.1 & 0.1 & 3.94 & 0.2 & 1.28 & 0.06 & 1.01 & 0.09 & 0.31 & 0 & 0.26 & 0.02 \\
\hline
\end{tabular}


that the PSE for number was significantly lower than the PSE for time. When collapsed across groups and blocks, the mean PSE for time (4.01) did not significantly differ from the geometric mean of 4.00 , while the mean PSE for number (3.86) was significantly below this value. None of the two-way interactions were significant, nor was the three-way interaction (all $F \mathbf{s}<3$ ).

Analysis of the DLs revealed a significant main effect of group $[F(1,10)=8.55, p<.05]$, a significant main effect of dimension $[F(1,10)=80.02, p<.01]$, and a significant group $\times$ dimension interaction $[F(1,10)=12.46$, $p<.05]$. The mean DL for number was significantly lower for the control animals than for the estradiol animals $[F(1,10)=12.39, p<.01]$, indicating that estradiol treatment significantly impaired sensitivity to number. However, although the difference in the mean DL for time between the two groups was also in the same direction, it was not statistically significant $[F(1,10)=3.66$, $p<.10]$. There was a significant main effect of block $[F(3,30)=4.74, p<.05]$ and a group $\times$ block interaction $[F(3,30)=7.70, p<.01]$. There was a significant decrease in the DL across blocks of testing for the estradiol group $[F(3,30)=12.05, p<.0001]$, but not for the control group $(F<1)$. This indicates that the sensitivity to time and number in the estradiol group increased as the time since exposure to estradiol increased. During the first block of testing, the mean DL, collapsed across time and number, was significantly lower for the control rats than for the estradiol rats $[F(1,10)=17.18, p<.01]$, indicating that treatment with estradiol significantly impaired sensitivity to time and number. However, the mean DL did not significantly differ between the two groups during the remaining blocks $[F \mathrm{~s}(1,10)=3.73,4.45$, and 0.53 , respectively]. The interaction between dimension and block was not significant $(F<1)$, nor was the threeway interaction $(F<2)$.

The WF data followed the same pattern as the DL data. Again, there was a significant main effect of group $[F(1,10)=14.44, p<.01]$, dimension $[F(1,10)=104.54$, $p<.01]$, and a significant group $\times$ dimension interaction $[F(1,10)=11.73, p<.05]$. The mean WF for number was significantly lower for the control animals than for the estradiol animals $[F(1,10)=20.29, p<.01]$. Although the difference in the mean WF for time between the two groups was in the same direction, it failed to achieve statistical significance $[F(1,10)=4.45, p=$ $.06]$. There was a significant main effect of block $[F(3,30)$ $=5.62, p<.01]$ and a significant group $\times$ block interaction $[F(3,30)=6.02, p<.01]$. There was a significant decrease in WF across blocks of testing for the estradiol group $[F(3,30)=11.25, p<.0001]$, but not for the control group $(F<1)$. During the first and second blocks of testing, the mean WF, collapsed across time and number, was significantly lower for the control rats than for the estradiol rats $[F(1,10)=26.41$ and 5.66 , respectively, $p<.05]$. However, the mean WF did not significantly differ between the two groups during the remaining two blocks $[F \mathrm{~s}(1,10)=2.89$ and 0.85 , respectively]. Neither the dimension $\times$ block, nor the group $x$ dimension $\times$ block interaction was significant $(F<2$ and $F<1$, respectively). These analyses show better discriminative control by time and number in the control group than in the group that had previously received estradiol injections. Discrimination improved across blocks of psychophysical testing for the estradiol animals, but was consistent across blocks for the control animals.

In summary, hormonal condition (estradiol, control) did not interact with dimension. That is, control by the temporal dimension was greater than control by the numerical dimension regardless of whether the functions under consideration were those of the estradiol rats or the control rats. The finding that time may be a more salient dimension than number is consistent with recently reported findings in both rats (Breukelaar \& DalrympleAlford, 1998, 1999) and pigeons (Roberts \& Boisvert, 1998). PSEs were at the geometric mean for time, but below the geometric mean for number. While a PSE at the geometric mean for time has frequently been reported (Breukelaar \& Dalrymple-Alford, 1999; Church \& Deluty, 1977; Fetterman \& Killeen, 1992; Maricq, Roberts, \& Church, 1981; Meck, 1983; Stubbs, 1976), a PSE at the geometric mean for number is less consistently found (Fetterman, 1993; Fetterman, Dreyfus, \& Stubbs, 1985; Martin-Iverson, Fibiger, \& Wilkie, 1988).

The major finding in Experiment 1 was that estradiol animals initially showed significantly flatter time and number functions than the control animals, and the slope of these functions became steeper as time since exposure to estradiol increased. PSEs were higher for time than for number, but they did not differ between groups or across blocks of testing. Estradiol administration seemed to decrease the discriminability of both time and number, as evidenced by the higher DLs and WFs for the estradiol group than for the control group.

Estradiol also decreased the animals' accuracy on the discrimination task and the number of trials completed per session prior to the start of psychophysical testing. The disruption in accuracy and reduction in trials completed both increased with continuing estradiol administration, but decreased when administration ceased. These effects of estrogen could be partially explained by estrogen's hypophagic effects. It has been demonstrated that gonadal hormones influence both food intake and bodyweight in rats (Wade, 1971, 1975). Estradiol injections have been consistently shown to reduce food intake, and the active site for these effects of estradiol seems to be the paraventricular nucleus of the hypothalamus (Butera, Xiong, Davis, \& Platania, 1996).

It would appear to be likely that the observed decrease in trials completed by the estradiol group was at least partially the result of these hypophagic effects of estradiol, since food was used as the reinforcer in the operant chamber. However, on the basis of these data, it was unclear whether the drop in accuracy observed in the estradiol group could also be attributed to this decrease in motivation. 


\section{EXPERIMENT 2}

Experiment 2 was designed to determine whether the effect of estradiol on the accuracy of time and number discriminations was simply a result of its effect on the motivation to work for food. Of the rats used in Experiment 1 , half were given access to food for $1 \mathrm{~h}$ prior to session initiation (the prefed group), and the other half were not (the non-prefed group). It was expected that the prefed group would experience a decrease in motivation similar to that induced by estradiol in Experiment 1 and display an associated decrease in the number of trials completed per session relative to the non-prefed group. If the prefed group also displayed lower discrimination accuracy relative to the non-prefed group, this would suggest that the drop in discrimination accuracy observed in the estradiol rats in Experiment 1 was due to estradiol's hypophagic effects. However, if the prefed group displayed equivalent accuracy to the non-prefed group despite showing a significant decrease in the number of trials completed, this would suggest that the drop in discrimination accuracy observed in the estradiol rats in Experiment 1 was not entirely due to a decrease in the motivation to work for food.

\section{Method}

\section{Subjects and Apparatus}

The subjects and apparatus were the same as those used in Experiment 1 .

\section{Procedure}

At the conclusion of Experiment 1, the rats were returned to their home cages for approximately 2 weeks. Following this, they were given 18 sessions of discrimination training in order to reestablish performance at baseline levels. The time/number discrimination task used in Experiment 2 was the same as the baseline training pro- cedure used in Experiment 1. Animals retained the assignment to the left or right lever on short/few trials that they learned during Experiment 1 and completed 160 trials or $120 \mathrm{~min}$ of training, whichever occurred first, each day for 18 consecutive days. Run times ranged between 55 and $120 \mathrm{~min}$. After the baseline sessions were completed, 18 prefeeding sessions were run. During these sessions, the 6 rats that composed the control group in Experiment 1 were designated the prefed group and were given access to food for $1 \mathrm{~h}$ before each experimental session. The amount of food made available to each prefed rat was weighed beforehand, and any remaining food was weighed $1 \mathrm{~h}$ later in order to ensure that the rats were eating. Rats that did not consume all available food were fed their remaining allotment after each experimental session. Nonprefed animals $(n=6)$ were fed only after the experimental session. After completion of the prefeeding sessions, the animals continued baseline training for an additional two sessions. No estrogen injections were given during any part of Experiment 2.

\section{Results and Discussion}

The discrimination accuracy and trials completed data were organized into three phases. The preexperimental phase consisted of the 2 days immediately prior to the prefeeding sessions; the experimental phase consisted of the 18 days during which the experimental group was prefed; and the postexperimental phase consisted of the 2 days immediately following the prefeeding sessions. The data obtained for trials completed per session for the prefed and non-prefed groups are presented in Figure 4. The non-prefed group completed a similar number of trials in each of the three phases; however, the prefed group completed markedly fewer trials during the experimental phase (prefeeding) than during either the preexperimental or postexperimental phase. An ANOVA of these data with group as the between-subjects factor and phase as the within-subjects factor was conducted. This revealed a significant main effect of phase $[F(2,20)=10.85, p<$ $.01]$ but not of group $(F<1)$. The phase $\times$ group inter-

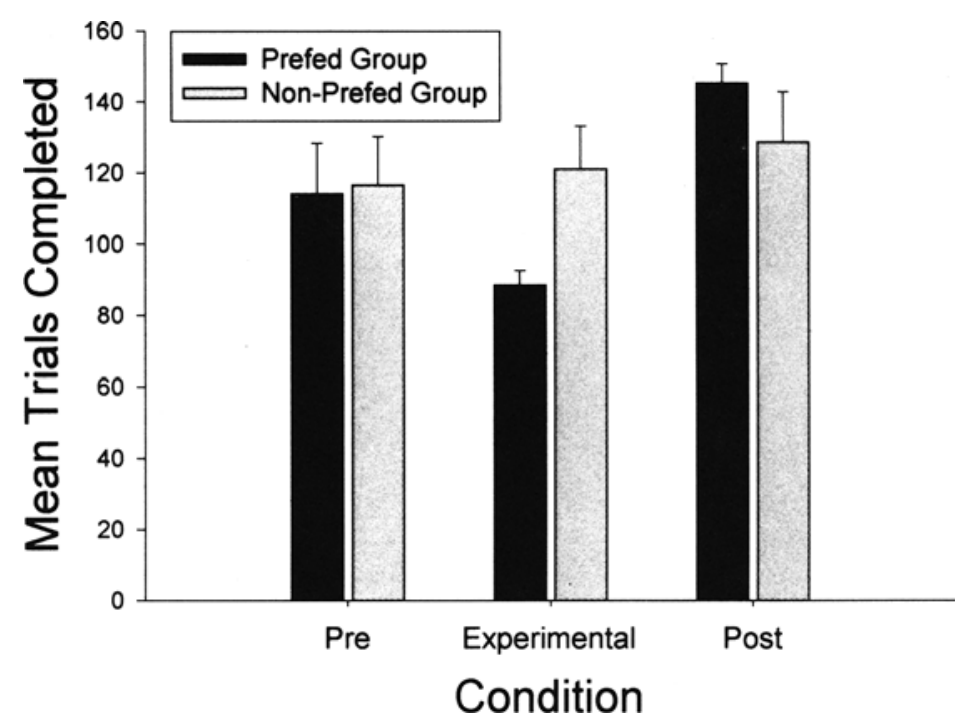

Figure 4. The mean number of trials completed by the prefed and the nonprefed groups during the preexperimental, experimental, and postexperimental phases of Experiment 2. 


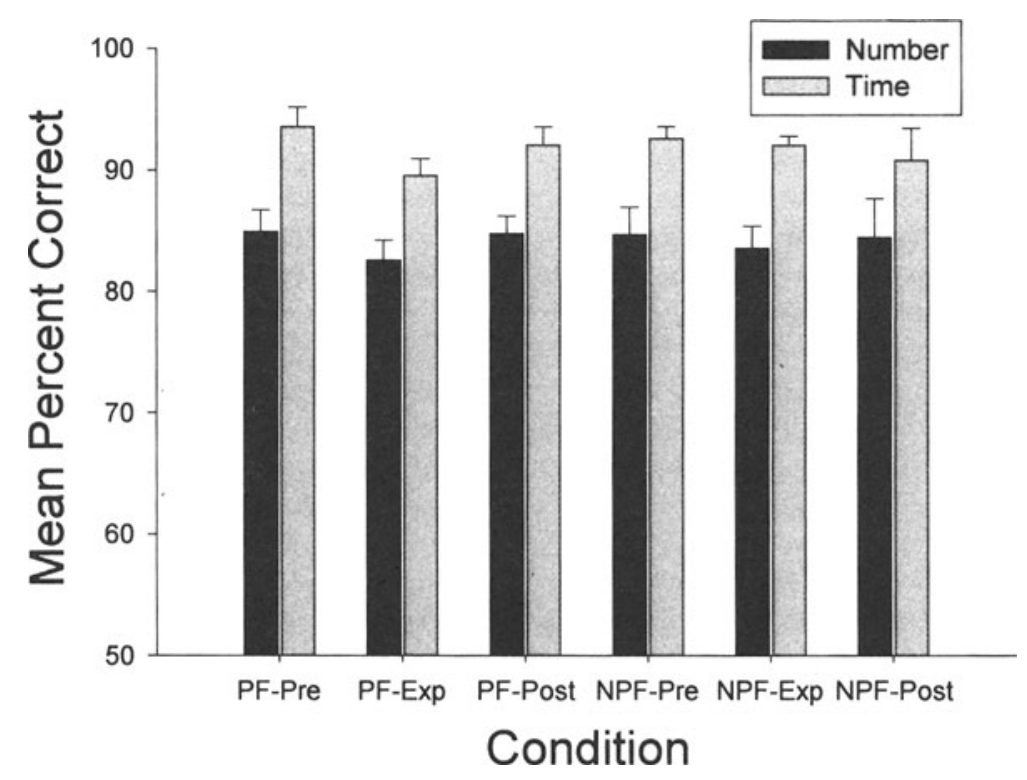

Figure 5. The mean percentage correct on time and number trials by the prefed (PF) and the non-prefed (NPF) groups during the preexperimental (Pre), experimental (Exp), and postexperimental (Post) phases of Experiment 2.

action was statistically significant $[F(2,20)=6.23, p<$ $.05]$ due to a significant effect of phase for the prefed group $[F(2,20)=16.33, p<.0005]$ but not for the control group $(F<1)$. Number of trials completed per session did not differ significantly between groups during the preexperimental and postexperimental phases $(F<1$ and $F<2$, respectively), but did differ during the experimental phase $[F(1,10)=6.73, p<.05]$. The finding that the prefed group completed fewer trials than the nonprefed group only during the experimental phase suggests, as expected, that the prefeeding procedure decreased the motivation to work for food.

The accuracy of time and number discriminations across the three phases for the prefed and non-prefed animals is shown in Figure 5. Accuracy was better on time trials than on number trials, as was the case in Experiment 1; however, there were no significant differences in accuracy either across phases or between groups, suggesting that the decrease in motivation produced by the prefeeding procedure did not result in a corresponding decrease in time or number discrimination accuracy. The data were analyzed using a mixed design ANOVA with group as the between-subjects factor and phase and dimension as the within-subjects factors. There was a significant main effect of dimension $[F(1,10)=51.87, p<.01]$, but not of phase or group ( $F<2$ and $F<1$, respectively). None of the two-way interactions reached statistical significance (all $F \mathrm{~s}<1$ ).

To summarize, the prefeeding procedure decreased motivation to work for food in such a way that animals given access to food before the experimental session completed fewer trials relative both to the non-prefed an- imals and to their own performance on the preexperimental and postexperimental sessions. There were no corresponding differences in discrimination accuracy, however, suggesting that prefeeding influenced motivation to perform the task without affecting accuracy on the task.

In Experiment 1, estradiol animals completed significantly fewer trials than did control animals during the injection period. However, these animals also had significantly lower accuracy on the task during this same period of time. The results of Experiment 2 suggest that decreased motivation to work for food, while sufficient to reduce the number of trials completed per session, is not sufficient to produce a deficit in the accuracy of discrimination of time and number. This would suggest that the drop in accuracy produced by estradiol in Experiment 1 might be independent of its hypophagic properties.

\section{GENERAL DISCUSSION}

In Experiment 1, a low physiological dose ( $8 \mu \mathrm{g} / \mathrm{kg})$ of estradiol benzoate was delivered in an injection regimen, which has previously been shown to increase the number of ChAT-like immunoreactive cells in the nucleus basalis magnocellularis of OVX rats (Gibbs, 1997). This hormone treatment resulted in a decrease in the number of trials completed per session during the injection period, coupled with a disruption in the accuracy of time and number processing postestrogen treatment. The former effect was attributed to the hypophagic effects of estradiol. In Experiment 2, a potential relationship between decreased motivation and disrupted discrimination accuracy was investigated. Animals given access to food for $1 \mathrm{~h}$ before 
each experimental session completed significantly fewer trials than did non-prefed animals; however, they did not show a corresponding decrease in discrimination accuracy. It appears that estradiol can have a negative impact on the discrimination of time and number that is independent of its hypophagic effects.

Two main types of behavioral procedures have been used in the study of timing in animals: the temporal bisection procedure and the peak-interval procedure. In the peak-interval procedure, animals are trained on a fixedinterval schedule to respond for food after a specified signaled interval has elapsed. On test trials, the duration of the signal is extended and no food is delivered. Because estrogen could have affected processes involved in the behavioral expression of timing rather than timing itself, the temporal bisection procedure was methodologically preferred over the peak-interval procedure for this study.

The psychophysical functions obtained for both time and number in the OVX control animals were consistent with findings from the previous literature on studies using intact male or female rats (Breukelaar \& DalrympleAlford, 1998; Meck \& Church, 1983). The psychophysical functions for the estradiol animals were not shifted horizontally, though they were shifted vertically in that they were initially flatter than the control functions. As time since exposure to estradiol increased, the functions became steeper and approached those of the control animals. PSEs did not differ significantly between the two groups and did not change across blocks of testing, suggesting that estrogen was not directly affecting the perception of time or number (i.e., clock processes). The estradiol animals did, however, have higher DLs and WFs than did control animals, and these values approached those of control animals as time since exposure to estradiol increased. This suggests that prior treatment with estrogen decreases the discriminability of both time and number signals, but that this effect becomes less severe over a period of several test days as the time since treatment increases.

Temporal processing was superior to numerical processing regardless of hormonal condition. This was evidenced both in the steeper slopes of the time functions and the lower values for time DLs and WFs relative to those for number. This is in contrast to the findings of Meck and Church (1983), but in agreement with the findings of Breukelaar and Dalrymple-Alford (1998), who have hypothesized that superior temporal processing occurs as a result of temporal information being considered before numerical information at the comparator phase of the internal clock model. As a result, when both temporal and numerical information are available, the temporal information will control responding and animals become less proficient at responding on the basis of number. This difference between time and number processing was found to be independent of the hormonal condition of the animal. The fact that time and number processing are similarly affected by estradiol provides additional support for the hypothesis that time and number pro- cessing involve the same internal mechanism (Meck \& Church, 1983).

The values of the PSEs obtained for time and number (as shown in Table 2) were similar to previous findings. The mean PSE for time was not significantly different from the geometric mean of 4.0, as was the case in the studies of Meck and Church (1983) and Breukelaar and Dalrymple-Alford (1998). The mean PSE for number, however, was significantly lower than the geometric mean. This is in contradiction to the results of Meck and Church and Breukelaar and Dalrymple-Alford (1998), who found no significant difference between PSEs for time and number, but it is supported by other studies showing the PSE for number to be significantly different from the geometric mean (Fetterman, 1993; Fetterman et al., 1985; Martin-Iverson et al., 1988).

One particularly interesting aspect of the results obtained in this study was that the effects of estrogen on both number of trials completed per session and discrimination accuracy persisted well after the hormone levels should have dropped to baseline. Although some studies have shown estrogen to be effective given as a single posttraining injection (Packard, 1998; Packard, Kohlmaier, \& Alexander, 1996; Packard \& Teather, 1997b), others have shown that optimal effects of estrogen may occur days or weeks after estrogen administration has ceased (Daniel, Fader, et al., 1997; Daniel, Spencer, \& Dohanich, 1997; Luine et al., 1998). The ability of estrogen to affect cognitive processing well after it has left the animal's system might help to explain the contradictory data that have resulted from investigations of spatial performance across the estrous cycle (Berry et al., 1997; Frye, 1995). It is possible that facilitatory or inhibitory effects of estrogen initiated during one phase of the cycle do not become behaviorally evident until later in the cycle when levels have changed. Because the estrous cycle is only 5-6 days in length, such long-term effects of estrogen could make consistent results difficult to obtain.

Although there is strong evidence for a role of the cholinergic system in estrogen's effects on cognitive processing (Dohanich et al., 1994; Fader et al., 1998; Packard et al., 1996), the results of this experiment suggest that estrogen does not interact with cholinergic components of the internal clock model. Differences in cholinergic functioning between estradiol and control groups should have been reflected in a horizontal shift of one group's function relative to the other's (see Meck, 1996). No such horizontal displacement of the functions was observed in this study. However, this does not rule out the possibility that estrogen may be interacting with the cholinergic system at some other stage of the cognitive processes necessary to make time and number discriminations. Further research will be necessary in order to ascertain whether the cholinergic system and not the dopaminergic system or some combination of neurotransmitter systems mediates the effects of estrogen on this task.

There are some potential limitations to the interpretation of the present results that require some comment. 
First, we were unable to measure serum estradiol in order to confirm that estradiol was present at expected levels during the administration period, and perhaps more importantly, that it was no longer present toward the end of the testing period. Behavioral evidence, however, suggests that this was the case. Decreases in the food intake of the estradiol-treated rats were noted within $48 \mathrm{~h}$ of estradiol administration. Similarly, food intake increased within $48 \mathrm{~h}$ of the last estradiol injection. Though not conclusive evidence for the assumed changes in estradiol levels, the fact that these same changes in feeding behavior were noted with the same rats and with different rats during subsequent studies (Ross \& Santi, unpublished data) suggests that feeding behavior may provide some useful information about estrogen levels.

A second factor that may potentially limit the interpretation of these results is the fact that estrogen administration began only after the rats had reached criterion on the task, while several studies of estrogen in spatial tasks have replaced estrogen either prior to or during acquisition (e.g. Daniel, Fader, et al., 1997; Fader et al., 1998). Since estrogen has been shown to have aversive properties in the conditioned taste avoidance paradigm (Ganesan \& Simpkins, 1990), it is possible that the performance deficits observed during estrogen administration were the result of state-dependent learning in that estrogen could have disrupted performance on a task that was acquired in its absence. This seems an unlikely explanation for the results, however, given that there are studies that have replaced estrogen after acquisition of a spatial task and still shown the hormone to have positive effects during testing (e.g., Dohanich et al., 1994; O’Neal, Means, Poole, \& Hamm, 1996).

A final factor that should be considered is the age of the animals at OVX. The rats in this study were OVX prepubertally (approximately 35 days of age), while the majority of researchers who have investigated the effects of estrogen on spatial tasks have used rats that were OVX as adults. Age at OVX has been shown to influence an animal's response to estrogen in physiological studies (Andrews, Advis, \& Ojeda, 1981; Wilkinson \& Bhanot, 1985). However, Daniel, Fader, et al. (1997) used rats that were also OVX prepubertally and still found an enhancing effect of estradiol on a spatial task. Interestingly, the enhancing effect found in this study was equally great when the animals received estradiol for 30 days prior to training as when the estradiol was administered during the 24 days of training. Taken together with the results of the present study, this might suggest that age at OVX could have some influence on the duration of estradiol's effects on cognition. Further research comparing effects of estrogen on cognitive tasks in rats that have been OVX at different ages would be useful in this respect.

The finding that estradiol disrupted performance on this task contradicts the results of numerous recent studies of the effects of estrogen on spatial processing in rats, several of which have shown facilitatory effects of estrogen (Daniel, Fader, et al., 1997; Daniel, Spencer, \&
Dohanich, 1997; Fader et al., 1998; Luine et al., 1998). These complex effects of estrogen on cognitive processing in animals raise questions about parallel effects in humans. Studies of cognitive processing in healthy women across the menstrual cycle suggest that estrogen may enhance verbal memory but inhibit spatial memory (Sherwin, 1994). However, these effects are of small magnitude and are not always consistent across studies (Epting \& Overman, 1998). Perhaps more relevant are the effects of estrogen on postmenopausal women with Alzheimer's disease. Estrogen replacement has been associated with lowered risk for the disease and has also been implicated in improved cognitive performance in postmenopausal women already suffering from Alzheimer's disease (Henderson, 1997). There are several potential mechanisms through which estrogen could exert these effects, including interactions with acetylcholine and other neurotransmitter systems, as well as neuroprotective effects (Mudd, Torres, Lopez, \& Montague, 1998). However, results of this study and others like it that have revealed negative effects of estrogen on cognitive processing in animals suggest that more research with different tasks and various patterns of estrogen administration will be necessary before estrogen can be considered as an effective treatment for Alzheimer's disease and other cognitive disorders.

\section{REFERENCES}

Andrews, W. W., Advis, J. P., \& OJeda, S. R. (1981). The maturation of estradiol-negative feedback in female rats: Evidence that the resetting of the hypothalamic "gonadostat" does not precede the first preovulatory surge of gonadotropins. Endocrinology, 109, 2022-2031.

BerRy, B., MCMAhaN, R., \& Gallagher, M. (1997). Spatial learning and memory at defined points of the estrous cycle: Effects on performance of a hippocampal-dependent task. Behavioral Neuroscience, 111, 267-274.

Breukelaar, J. W. C., \& Dalrymple-Alford, J. C. (1998). Timing ability and numerical competence in rats. Journal of Experimental Psychology: Animal Behavior Processes, 24, 84-97.

BreukelaAr, J. W. C., \& Dalrymple-Alford, J. C. (1999). Effects of lesions to the cerebellar vermis and hemispheres on timing and counting in rats. Behavioral Neuroscience, 113, 78-90.

Butera, P. C., Xiong, M., Davis, R. J., \& Platania, S. P. (1996). Central implants of dilute estradiol enhance the saticty effect of CCK-8. Behavioral Neuroscience, 110, 823-830.

Church, R. M. (1984). Properties of the internal clock. In J. Gibbon \& L. Allen (Eds.), Timing and time perception (Annals of the New York Academy of Sciences, Vol. 423, pp. 566-582). New York: New York Academy of Sciences.

Church, R. M., \& Deluty, M. Z. (1977). Bisection of temporal intervals. Journal of Experimental Psychology: Animal Behavior Processes, 3, 216-228.

Daniel, J. M., Fader, A. J., Spencer, A. L., \& Dohanich, G. P. (1997). Estrogen enhances performance of female rats during acquisition of a radial arm maze. Hormones \& Behavior, 32, 217-225.

Daniel, J. M., Spencer, A. L., \& Dohanich, G. P. (1997). Effects of prior estrogen exposure on radial maze acquisition in female rats. Society for Neuroscience Abstracts, 23, 2124.

Dohanich, G. P., Fader, A. J., \& Javorsky, D. J. (1994). Estrogen and estrogen-progesterone treatments counteract the effect of scopolamine on reinforced T-maze alternation in female rats. Behavioral Neuroscience, 108, 988-992.

EPting, L. K., \& Overman, W. H. (1998). Sex-sensitive tasks in men and women: A search for performance fluctuations across the menstrual cycle. Behavioral Neuroscience, 112, 1304-1317. 
FADER, A. J., Hendricson, A. W., \& Dohanich, G. P. (1998). Estrogen improves performance of reinforced T-maze alternation and prevents the amnestic effects of scopolamine administered systemically or intrahippocampally. Neurobiology of Learning \& Memory, 69, 225-240.

Fetterman, J. G. (1993). Numerosity discrimination: Both time and number matter. Journal of Experimental Psychology: Animal Behavior Processes, 19, 149-164.

Fetterman, J. G., Dreyfus, L. R., \& Stubbs, D. A. (1985). Scaling of response-based events. Journal of Experimental Psychology: Animal Behavior Processes, 11, 388-404.

Fetterman, J. G., \& Killeen, P. R. (1992). Time discrimination in Columbia livia and Homo sapiens. Journal of Experimental Psychology: Animal Behavior Processes, 18, 80-94.

FILLIT, H. (1994). Estrogens in the pathogenesis and treatment of Alzheimer's disease in postmenopausal women. In V. N. Luise \& C. F. Harding (Eds.), Hormonal restructuring of the adult brain: Basic and clinical perspectives (Annals of the New York Academy of Sciences, Vol. 743, pp. 233-239). New York: New York Academy of Sciences.

Fink, G., Sumner, B. E. H., McQueEn, J. K., Wilson, H., \& Rosie, R. (1998). Sex steroid control of mood, mental state and memory. Clinical \& Experimental Pharmacology \& Physiology, 25, 764-775.

FrYE, C. A. (1995). Estrus-associated decrements in a water maze task are limited to acquisition. Physiology \& Behavior, 57, 5-14.

Gallistel, C. R. (1990). The organization of learning. Cambridge, MA: MIT Press.

GanesAn, R., \& Simpkins, J. W. (1990). The role of conditioned taste aversion in the suppression of food intake by estradiol. Physiology \& Behavior, 48, 647-652.

Gibbon, J., Malapani, C., Dale, C. L., \& Gallistel, C. R. (1997). Towards a neurobiology of temporal cognition: Advances and challenges. Current Opinion in Neurobiology, 7, 170-184.

GiBBS, R. B. (1997). Effects of estrogen on basal forebrain cholinergic neurons vary as a function of dose and duration of treatment. Brain Research, 757, 10-16.

GibBS, R. B., \& AGgarwal, P. (1998). Estrogen and basal forebrain cholinergic neurons: Implications for brain aging and Alzheimer's disease-related cognitive decline. Hormones \& Behavior, 34, 98-111.

GibBs, R. B., Burke, A. M., \& Johnson, D. A. (1998). Estrogen replacement attenuates effects of scopolamine and lorazepam on memory acquisition and retention. Hormones \& Behavior, 34, 112-125.

HENDERSON, V. W. (1997). Estrogen, cognition, and a woman's risk of Alzheimer's disease. American Journal of Medicine, 103(3A), $11 \mathrm{~S}-18 \mathrm{~S}$.

Luine, V.N., Richards, S. T., WU, V. Y., \& Beck, K. D. (1998). Estradiol enhances learning and memory in a spatial memory task and effects levels of monoaminergic neurotransmitters. Hormones \& $\mathrm{Be}$ havior, 34, 149-162.

MaricQ, A. V., Roberts, S., \& Church, R. M. (1981). Methamphetamine and time estimation. Journal of Experimental Psychology: Animal Behavior Processes, 7, 18-30.

Martin-Iverson, M. T., Fibiger, H. C., \& Wil.kie, D. M. (1988). Alteration in the perception of food quantity by rats induced by manipulations of hunger and food sweetness. Learning \& Motivation, 19, 44-65.

McEwen, B. S., Alves, S. E., Bulloch, K., \& Weiland, N. G. (1997). Ovarian steroids and the brain: Implications for cognition and aging. Neurology, 48(S7), S8-S15.

MECK, W. H. (1983). Selective adjustment of the speed of internal clock and memory processes. Journal of Experimental Psychology: Animal Behavior Processes, 9, 171-201.

MECK, W. H. (1996). Neuropharmacology of timing and time perception. Cognitive Brain Research, 3, 227-242.

MECK, W. H., \& ChURCH, R. M. (1983). A mode control model of count- ing and timing processes. Journal of Experimental Psychology: Animal Behavior Processes, 9, 320-334.

MecK, W. H., ChurCh, R . M., \& GibBon, J. (1985). Temporal integration in duration and number discrimination. Journal of Experimental Psychology: Animal Behavior Processes, 11, 591-597.

Mudd, L. M., Torres, J., Lopez, T. F., \& MonTAGue, J. (1998). Effects of growth factors and estrogen on the development of septal cholinergic neurons from the rat. Brain Research Bulletin, 45, 137-142.

O'Neal, M. F., Means, L. W., Poole, M. C., \& Hamm, R. J. (1996). Estrogen affects performance of ovariectomized rats in a two-choice water-escape working memory task. Psychoneuroendocrinology, 21, 51-65.

PaCKard, M. G. (1998). Posttraining estrogen and memory modulation. Hormones \& Behavior, 34, 126-139.

Packard, M. G., Kohlmaier, J. R., \& Alexander, G. M. (1996). Posttraining intrahippocampal estradiol injections enhance spatial memory in male rats: Interaction with cholinergic systems. Behavioral Neuroscience, 110, 626-632.

PaCkard, M. G., \& TEather, L. A. (1997a). Intra-hippocampal estradiol infusion enhances memory in ovariectomized rats. NeuroReport, 8, 3009-3013.

Packard, M. G., \& Teather, L. A. (1997b). Posttraining estradiol injections enhance memory in ovariectomized rats: Cholinergic blockade and synergism. Neurobiology of Learning \& Memory, 68, 172-188.

ROBERTS, W. A., \& BoISVERT, M. J. (1998). Using the peak procedure to measure timing and counting processes in pigeons. Journal of $E x-$ perimental Psychology: Animal Behavior Processes, 24, 416-430.

SHERWIN, B. B. (1994). Estrogenic effects on memory in women. In V. N. Luise \& C. F. Harding (Eds.), Hormonal restructuring of the adult brain: Basic and clinical perspectives (Annals of the New York Academy of Sciences, Vol, 743, pp. 213-231). New York: New York Academy of Sciences.

Singh, M., Meyer, E. M., Millard, W. J., \& Simpkins, J. W. (1994). Ovarian steroid deprivation results in a reversible learning impairment and compromised cholinergic function in female SpragueDawley rats. Brain Research, 644, 305-312.

Stackman, R. W., Blasberg, M. E., Langan, C. J., \& Clark, A. S. (1997). Stability of spatial working memory across the estrous cycle of Long-Evans rats. Neurobiology of Learning \& Memory, 67, 167-171.

STUBBS, D. A. (1976). Scaling of stimulus duration by pigeons. Journal of the Experimental Analysis of Behavior, 26, 15-25.

WADE, G. N. (1971). Gonadal hormones and behavioral regulation of body weight. Physiology \& Behavior, 8, 523-534.

WADE, G. N. (1975). Some effects of ovarian hormones on food intake and body weight in female rats. Journal of Comparative \& Physiological Psychology, 88, 183-193.

WARREN, S. G., \& JURASKA, J. M. (1997). Spatial and nonspatial learning across the rat estrous cycle. Behavioral Neuroscience, 111, 259-266.

WILKINSON, M., \& BHANOT, R. (1985). Infantile ovariectomy potentiates the stimulatory effect of oestrogen/progesterone on $\mathrm{LH}$ secretion in the rat. Journal of Endocrinology, 106, 133-139.

WoOLley, C. S. (1998). Estrogen-mediated structural and functional synaptic plasticity in the female rat hippocampus. Hormones $\& B e$ havior, 34, 140-148.

Woolley, C. S., Gould, E., Frankfurt, M., \& McEwen, B. S. (1990). Naturally occurring fluctuation in dendritic spine density on adult hippocampal pyramidal neurons. Journal of Neuroscience, 10, 40354039.

(Manuscript received July 28, 1999; revision accepted for publication December 23, 1999.) 